\title{
Simulations of hybrid long-range plasmon modes with application to $90^{\circ}$ bends
}

\author{
Aloyse Degiron, ${ }^{1}$ Claudio Dellagiacoma, ${ }^{2}$ James G. McIlhargey, ${ }^{3}$ Gennady Shvets, ${ }^{3}$ Olivier J. F. Martin, ${ }^{2}$ \\ and David R. Smith ${ }^{1, *}$ \\ ${ }^{1}$ Department of Electrical and Computer Engineering, Duke University, Box 90291, Durham, \\ North Carolina 27708, USA \\ ${ }^{2}$ Nanophotonics and Metrology Laboratory, Swiss Federal Institute of Technology Lausanne, EPFL-STI-ITOP-NAM, \\ Station 11, CH-1015 Lausanne, Switzerland \\ ${ }^{3}$ Department of Physics, The University of Texas at Austin, Austin, Texas 78712, USA \\ *Corresponding author: drsmith@ee.duke.edu
}

Received May 8, 2007; revised June 12, 2007; accepted June 23, 2007;

posted July 3, 2007 (Doc. ID 82798); published August 2, 2007

\begin{abstract}
We perform rigorous simulations of hybrid long-range modes guided by a central metal core and a twodimensional dielectric slab. We show that these modes are subject to fewer limitations than conventional long-range plasmon modes in terms of field confinement and guiding performance. These hybrid modes may offer substantial improvements for integrated plasmonic components, as illustrated here by the consideration of $90^{\circ}$ bends. (C) 2007 Optical Society of America
\end{abstract}

OCIS codes: $240.6680,130.2790,130.3120$.

Surface plasmon polaritons (SPPs) are electromagnetic-electronic waves propagating at the interface between a metal and a dielectric [1]. SPPs are associated with strongly enhanced local fields that can exhibit spatial variation on a scale much smaller than the photon wavelength. This particular aspect of SPPs renders them attractive for the development of photonic components whose size is not limited by the diffraction limit of light [2]. However, SPP modes with subdiffraction confinement propagate only over distances ranging from micrometers in the visible to hundreds of micrometers in the infrared, because a substantial part of the energy is contained in the metal-an extremely absorptive medium at those frequencies $[1,3,4]$. To reduce the losses by absorption, the field confinement must be considerably relaxed [4]: for example, thin metal films and metal strips support long-range SPPs that propagate as far as centimeters, but the lateral extension of these modes reaches several wavelengths [5-15].

Recently, several studies have shown that the properties of long-range SPPs propagating along infinitely wide metal films can be tailored by constraining their field in a two-dimensional dielectric waveguide [12-14]. Here we generalize this approach by considering the case of arbitrary curved metal strips embedded in a thin dielectric slab. We show numerically that the long-range SPPs are hybridized by total internal reflection and that, although the general trade-off between loss and propagation distance still occurs for these modes, the electromagnetic fields can be localized to significantly smaller volumes for the same propagation distance when compared with long-range SPPs.

Our numerical method is based on finding the eigenmodes of the strip geometry in either an infinite- or a finite-thickness dielectric region at the wavelength $\lambda=1550 \mathrm{~nm}$. To simplify the calculations, we limit our investigations to straight and uniformly bent waveguides and investigate their modes in Car- tesian and cylindrical coordinates, respectively (Fig. 1). The electric field of the mode can be formally written as $E=E_{0}(x, y) \exp \left(i \beta_{z} z\right)$ for the straight waveguides and $E=E_{0}(r, y) \exp \left(i \beta_{\theta} \theta\right)$ for the bends, where $E_{0}$ is the field distribution along the waveguide cross section and $\beta_{z}$ (in inverse meters) and $\beta_{\theta}$ (in inverse radians) are the complex propagation constants. With the field dependence on the propagation direction assumed, the strip waveguides can be fully characterized by performing a finite-element analysis of their two-dimensional cross section. This task was carried out by using Comsol Multiphysics, a commercial package capable of calculating the modes by solving an integral form of Maxwell's equations in which the mode propagation constant is the eigenvalue [16]. In the following, we focus on the fundamental long-
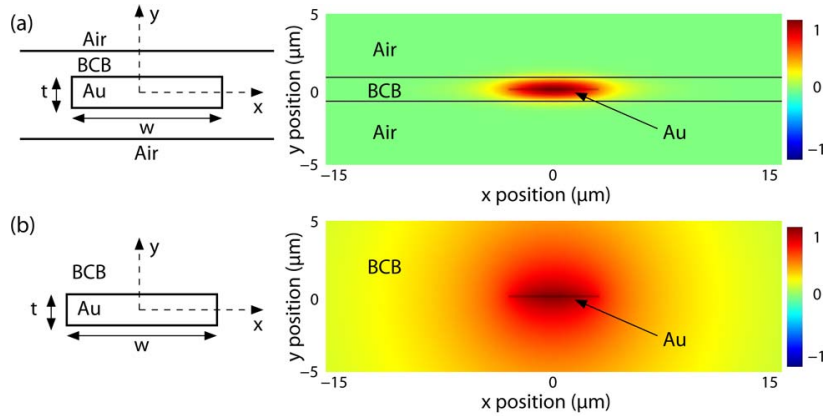

(c)

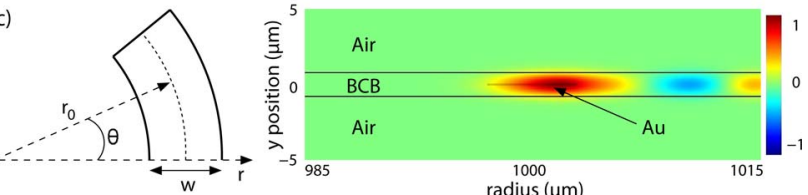

Fig. 1. (Color online) Geometry and $E_{y}$ field component for the fundamental long-range mode of (a) a straight Au strip of rectangular cross section $(w=6 \mu \mathrm{m}, t=10 \mathrm{~nm})$ in a BCB layer of thickness $1.6 \mu \mathrm{m}$; (b) the same Au strip in an infinite volume of BCB; (c) a curved Au strip of rectangular cross section $\left(w=6 \mu \mathrm{m}, t=10 \mathrm{~nm}, r_{0}=1 \mathrm{~mm}\right)$ in a BCB layer of thickness $1.6 \mu \mathrm{m}$. 
range mode of the structures, but we note that higher-order modes might also exist at the same wavelength, depending on the dimensions of the configuration.

Figure 1(a) shows the first structure under consideration. It consists of a straight rectangular $\mathrm{Au}$ strip embedded in a $1.6 \mu \mathrm{m}$ thick layer of benzocyclobutene (BCB) and surrounded by air on both sides. The Au strip is $6 \mu \mathrm{m}$ wide, $10 \mathrm{~nm}$ thick, and has a complex permittivity of $-114+11 i$ at $\lambda=1550 \mathrm{~nm}$ [17]; the index of refraction of $\mathrm{BCB}$ is 1.535 at the same wavelength [12]. Because of these material parameters, the structure of Fig. 1(a) can be considered a combination of a plasmonic waveguide (the metal strip) and a two-dimensional dielectric waveguide (the BCB layer) in which the fields are constrained by total internal reflection. The hybrid nature of the waveguide is clearly illustrated in Figs. 1(a) and 1(b), which reveal that the field pattern of the fundamental long-range mode is similar to that of a strip in an infinite environment, except that the fields are frustrated vertically at the BCB-air interfaces. Figure 1(a) suggests that the hybrid mode remains predominantly guided by the metal core rather than by the BCB because its electric field is bound to and centered on the strip, with an amplitude maximum at the metal surface. To further verify this assumption, we have also plotted the electric field pattern when the strip is bent with a radius of curvature $r_{0}=1 \mathrm{~mm}$. Figure 1(c) shows that the mode has become asymmetric but that it is located around-and thus guided by-the metal strip.

However, the fundamental hybrid mode of Fig. 1(c) is not strictly bound to the metal, because it emits light into the BCB layer, as evidenced by the radiation pattern on the external side of the curvature. To quantify the effect of radiation losses on our hybrid mode, we have computed the mode propagation length $l=r_{0} /\left[2 \operatorname{Im}\left(\beta_{\theta}\right)\right]$ as a function of the radius of curvature $r_{0}$ [Fig. 2(a)]. For large values of $r_{0}$, the propagation length reaches approximately $4.5 \mathrm{~mm}$ for all bends-a distance much larger than shortrange SPPs [1]. In this limit, the mode is virtually the same as the nonradiative mode of the straight waveguide of Fig. 1(a), and therefore the propagation length is determined entirely by the absorption losses in the metal. For sharper bends, the propagation distance drops rapidly because the mode becomes increasingly radiative, as already shown by the field pattern of Fig. 1(c).

Figure 2(b) shows the corresponding loss $L$ for hybrid $90^{\circ}$ bends as a function of $r_{0}$, where $L$ is defined as the ratio in decibels of the intensities at the output and input of a bend. It can be seen that there occurs an optimum radius of curvature that varies with the configuration of strip and dielectric thickness, for which the losses are minimized. This characteristic behavior has been predicted by Berini for metallic strips embedded in an infinite medium and results from a trade-off between the radiation losses (which increase as the bend becomes sharper) and the damping by absorption (which increase as the bend becomes longer) [18]. For the fundamental hybrid mode

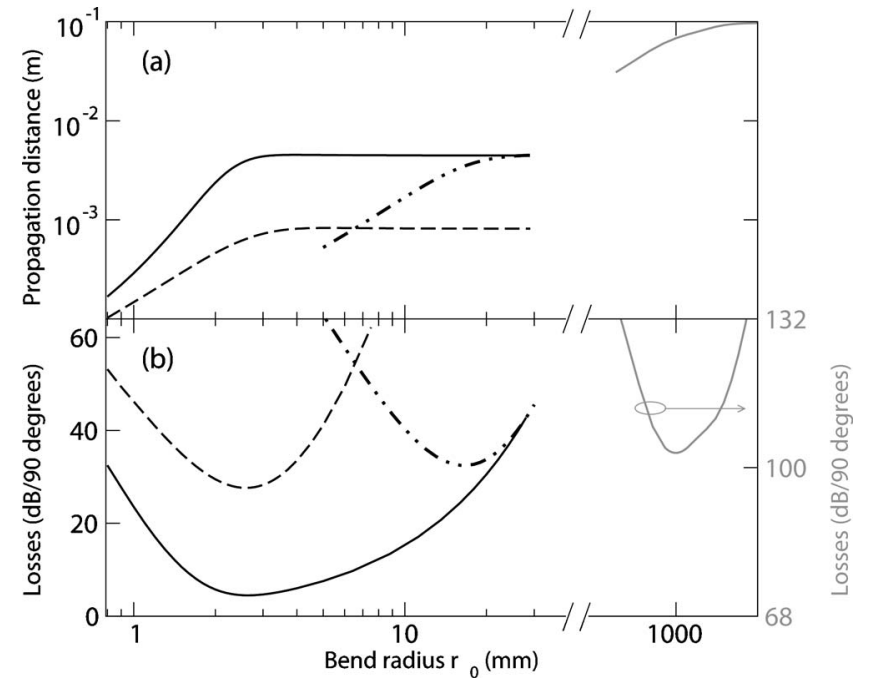

Fig. 2. (a) Propagation distance of the fundamental longrange mode as a function of $r_{0}$. Black curve, Au strip $(w=6 \mu \mathrm{m}, t=10 \mathrm{~nm})$ in a BCB layer of thickness $1.6 \mu \mathrm{m}$; grey curve, same strip, but in an infinite volume of $\mathrm{BCB}$; dashed-dotted curve, Au strip ( $w=6 \mu \mathrm{m}, t=19.918 \mathrm{~nm})$ in an infinite volume of BCB; dashed curve, $\mathrm{Au}$ strip $(w=6 \mu \mathrm{m}, t=32.5 \mathrm{~nm})$ in an infinite volume of BCB. (b) Total losses after the mode has turned a $90^{\circ}$ angle for the same structures.

studied here, an optimized bend radius of $r_{\mathrm{opt}}$ $=2.4 \mathrm{~mm}$ is found.

As a comparison, Fig. 2 also plots the mode characteristics when the strip is embedded in an infinite volume of BCB. In this case, the field associated with the SPP mode fully extends in the dielectric [Fig. 1(b)], and therefore the propagation length is much higher, reaching $10 \mathrm{~cm}$ when the bend tends to a straight waveguide. On the other hand, Fig. 2(b) shows that $r_{\text {opt }}>1 \mathrm{~m}$ and $L>100 \mathrm{~dB}$ for a $90^{\circ}$ bend, thus indicating that the mode is so weakly bound to the strip that it cannot be guided by other structures than (almost) straight waveguides. This being said, long-range SPPs supported by strips in an infinite dielectric medium are not always as poorly guided as in this example. In particular, the field confinement can be substantially improved by simply increasing the strip thickness [6], in which case the mode propagates over smaller distances but rounds sharper bends [18]. However, the relationship between field confinement, propagation distance, and robustness with regards to bending is less favorable than for the hybrid mode. For example, Fig. 2 shows that, when the strip thickness reaches $20 \mathrm{~nm}$, the maximum propagation distance of the long-range SPP is the same as for the hybrid mode but that $r_{\text {opt }}$ and $L$ are much higher. To reduce the optimum radius of curvature down to the value of the hybrid case, the field confinement-and therefore the strip thicknessmust be further increased. We found that the strip should be $32.5 \mathrm{~nm}$ thick to meet this condition; however, the corresponding losses are $30 \mathrm{~dB}$ larger than for the hybrid case because the mode is entirely damped when the total length of this waveguide exceeds $800 \mu \mathrm{m}$ (Fig. 2). 
To complete these comparisons, it is interesting to examine the field confinement of the different modes considered here. The electric field amplitude along the vertical axis of symmetry for each of the straight waveguides is shown in Fig. 3. The confinement of the hybrid mode is far superior to the others, including the thickest strip for which the long-range mode propagates only over $800 \mu \mathrm{m}$. In fact, by quantifying the confinement as the length over which the field decreases down to 1/e of its maximum value, the hybrid mode is the only mode exhibiting a slight subwavelength confinement.

In summary, the hybrid SPP mode exhibits the key features of good confinement along with excellent guiding ability-that is, the SPP propagates with minimal absorption. The origin of the improved performance of the hybrid modes can be understood by a closer examination of the field patterns of Fig. 3. For conventional SPPs, the electromagnetic field decays exponentially on both sides of the metal surface and therefore the local energy density has always the same gradient profile-it is extremely high in the vicinity of the metal surface and much weaker everywhere else. Because of this characteristic distribution, the slightest reduction of the mode lateral size leads to a dramatic rise of the energy density inside and just above the metal, and therefore the losses by absorption in the metal increase at a large pace. In contrast, the fields associated with the hybrid mode are frustrated by total internal reflection; so the resulting modal pattern is more akin to a Gaussian. In this case, the gradient of the local energy density is substantially weaker than for conventional SPPs, and thus the fundamental limitations related to the exponential decay of the electric field away the surface have been leveraged.

It should be noted that the field of the hybrid mode exhibits a small discontinuity at the BCB-air inter-

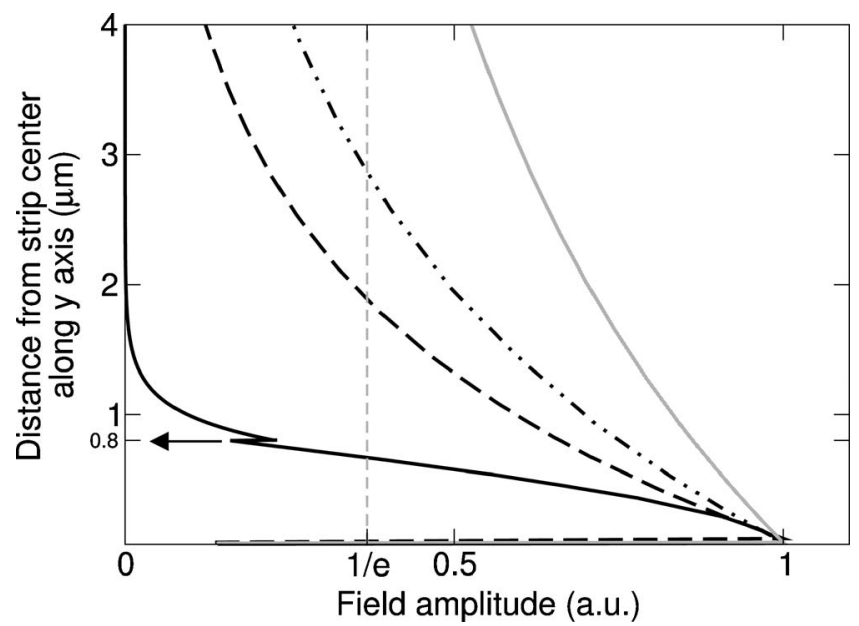

Fig. 3. Vertical field profile for the modes examined in Fig. 2 (the same conventions are used). The arrow shows the position of the BCB-air interface for the hybrid mode. This graph only considers the straight waveguide case. face and then decays exponentially in the air region (Fig. 3). We find that as the BCB layer becomes thinner, this exponential tail eventually becomes extremely large so that the field confinement and related benefits are lost. Therefore the BCB thickness must be adjusted carefully to optimize the performance of the hybrid waveguide. The BCB thickness chosen in the above examples is close to the optimum value for the metal strips and wavelength considered here. Although we only investigated the case of $10 \mathrm{~nm}$ thick metal strips, which are technically challenging to fabricate [9,17], hybrid long-range SPPs can also be obtained for thicker metal cores $(t>20 \mathrm{~nm})$ as long as their electromagnetic field remains constrained by a dielectric slab.

In conclusion, we have suggested a route to leverage an important limitation associated with longrange plasmons, namely, the trade-off among field confinement, guiding performances, and losses. We see that this limitation is a direct consequence of the mode profile and that it is therefore possible to push it back by perturbing the fields associated with the mode.

This work was performed under a Multiple University Research Initiative supported by the Air Force Office of Scientific Research (contract FA9550-04-10434).

\section{References}

1. H. Raether, Surface Plasmons (Springer-Verlag, 1988).

2. W. L. Barnes, A. Dereux, and T. W. Ebbesen, Nature 424, 824 (2003).

3. W. L. Barnes, J. Opt. A 8, S87 (2006).

4. P. Berini, Opt. Express 14, 13030 (2006).

5. D. Sarid, Phys. Rev. Lett. 47, 1927 (1981).

6. P. Berini, Phys. Rev. B 61, 10484 (2001).

7. P. Berini, Phys. Rev. B 63, 125417 (2001).

8. R. Charbonneau, P. Berini, E. Berolo, and E. LisickaShrzek, Opt. Lett. 52, 844 (2000).

9. P. Berini, R. Charbonneau, N. Lahoud, and G. Mattiussi, J. Appl. Phys. 98, 043109 (2005).

10. R. Charbonneau, C. Scales, I. Breukelaar, S. Fafard, N. Lahoud, G. Mattiussi, and P. Berini, J. Lightwave Technol. 24, 477 (2006).

11. T. Nikolajsen, K. Leosson, and S. I. Bozhevolnyi, Appl. Phys. Lett. 85, 5833 (2004).

12. T. Nikolajsen, K. Leosson, I. Salakhutdinov, and S. I. Bozhevolnyi, Appl. Phys. Lett. 82, 668 (2003).

13. A. Boltasseva, T. Nikolajsen, K. Leosson, K. Kjaer, M. S. Larsen, and S. I. Bozhevolnyi, J. Lightwave Technol. 23, 413 (2005).

14. J. Guo and R. Adato, Opt. Express 14, 12409 (2006).

15. A. Degiron and D. R. Smith, Opt. Express 14, 1611 (2006).

16. C. Dellagiacoma, O. J. F. Martin, A. Degiron, J. J. Mock, and D. R. Smith, "Simulations of integrated optical circuits combining dielectric and plasmonic elements," (manuscript in preparation).

17. P. B. Johnson and R. W. Christy, Phys. Rev. B 6, 4370 (1972).

18. P. Berini, Opt. Express 14, 2365 (2006). 